\title{
Red Ear Syndrome and migraine: case report and review of this peculiar association
}

\author{
Juliana Baleki Borri' (iD, Hilton Mariano da Silva Junior ${ }^{1,2}$ \\ ${ }^{1}$ Pontifícia Universidade Católica de Campinas, Campinas, São Paulo, Brazil; \\ ${ }^{2}$ Hospital Municipal de Campinas Doutor Mário Gatti, Campinas, São Paulo, Brazil
}

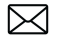

Juliana Baleki Borri

juliana.baleki@gmail.com

Edited by:

Marcelo Moraes Valença

Keywords:

Migraine

migraine disorders

Erythema

Pain

Red ear syndrome

\begin{abstract}
Introduction

The "Red Ear Syndrome" (RES) is a rare condition (about 100 cases were published in 25 years). It is characterized by episodes of hyperemia of the ear associated with burning pain. Although the association of this syndrome and primary headaches is contemplated in literature, its etiology and treatments are still poorly defined.

The aim of this paper is to report a case of RES related to migraine and possible pathophysiologic mechanisms.

Case Report

A 31 -year-old woman presented with stabbing pain and marked erythema and edema of the right ear accompanied by burning and local hyperhidrosis. These attacks lasted 2 hours on average, and either occurred spontaneously or were associated with migraine without aura. The only means of relief during the attacks was cooling the local with ice. Extensive laboratory-chemical, microbiological, ear-nose-throat, clinical and neurological examinations, magnetic resonance of the brain and cervical spine were unremarkable.

\section{Conclusion}

Uncertainty about the etiology of this syndrome is an obstacle to treatment. The frequent relationship between RES and migraine suggests that is necessary to investigate the syndrome in migraineurs. Furthermore, new reports about this disorder are important to increase the knowledge of physicians, to reduce the delay in diagnosis and suffering of patients.
\end{abstract}




\section{Introduction}

The "Red Ear Syndrome" (RES) is a rare condition. Its etiology and treatments are still poorly defined. It is characterized by episodes of hyperemia of the ear associated with burning pain. RES can be associated with primary headaches. This relationship was first described in 1996, when Lance published a series of cases on the syndrome, and two patients had both RES and migraine. 'From this, other reports emerged and one of the hypotheses is that the red ear phenomenon shares pathophysiological mechanisms with migraine.

This syndrome can be classified into two forms. The idiopathic form is often associated with primary headaches, such as migraine and trigeminal autonomic headache. Nevertheless, it may occur spontaneously and is more frequent in young people. The secondary form, on the other hand, is related to structural disorders, such as lesions and irritations of $\mathrm{C} 3$ and $\mathrm{C} 4$ roots or temporomandibular dysfunction and are more frequent in older patients. ${ }^{2,3}$

\section{Case Report}

We report a 31-year-old female patient, who had been presenting for 2 years with acute pain, edema and accentuated erythema in the right ear pavilion (Figure 1), occasionally accompanied by hyperhidrosis, and local burning. These attacks lasted 2 hours on average, they occurred either spontaneously or were induced by emotional stress or direct contact with heat. All crises were associated with hyperemia and redness of the ear, which started a few minutes after the pain, and normalized with its resolution. During attacks, cooling was the only means of relief.

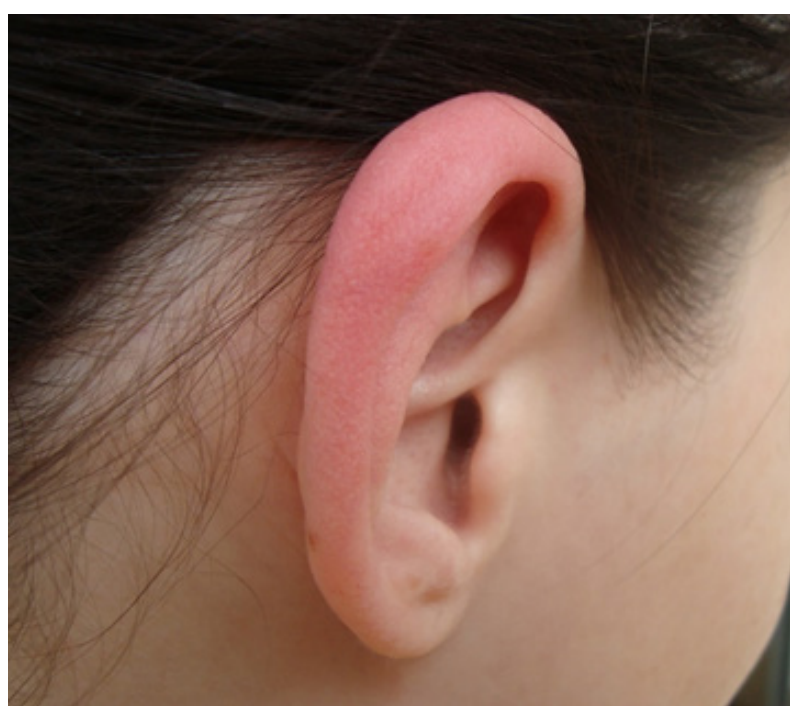

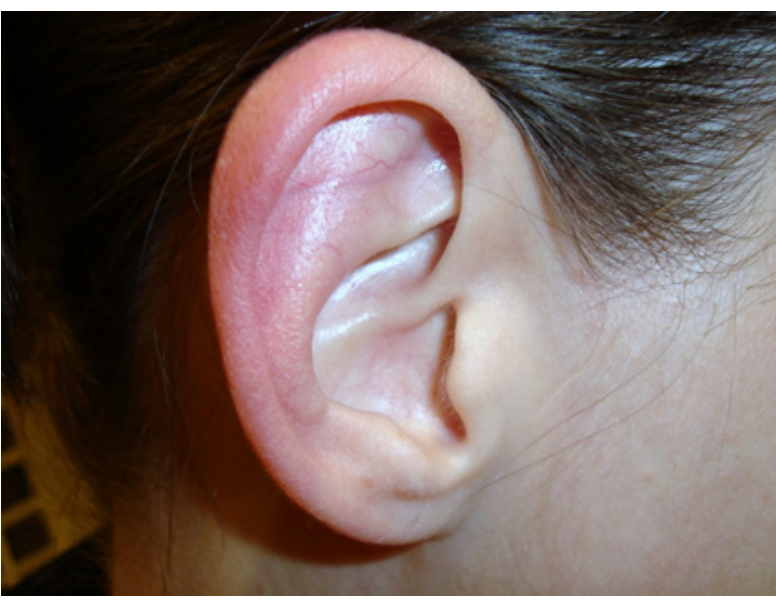

Figure 1. Edema and accentuated erythema in the right ear pavilion.

The patient has also complained of migraine without aura since adolescence, and reported a clear association between the syndrome and some migraine attacks. The patient used flunarizine for both headache and red ear, but treatment was interrupted due to side effects.

Extensive laboratory-chemical, microbiological, ear-nosethroat, clinical and neurological examinations, as well as magnetic resonance of the brain and cervical spine were unremarkable.

\section{Discussion}

\section{Epidemiology}

According to the description of signs and symptoms, associated to normal exams and absence of structural lesions, the patient fits into the classification of primary RES. This syndrome appears to be more prevalent in women, in the adult population, and is more prevalent than imagined in the pediatric population, mostly male. ${ }^{4.8}$ The median age of RES onset is 44 years old, ranging from 4 to 92 years. $^{9}$

Due to the scarcity of case reports, prevalence and incidence of RES are unknown. Notwithstanding, Raieli et al. ${ }^{5}$, in a study with 92 participants aged 6 to 18 years, reported that $24 \%$ of migraneurs patients had RES. That is a relatively high number for such an unknown phenomenon. This suggests that the syndrome should be investigated in migraine patients. Furthermore, RES may be less rare than expected, especially occurring during primary headache, 
and considered as a symptom. ${ }^{8}$

\section{Clinical presentation}

Episodes may occur concomitantly with primary headache attacks, more common with migraine, or occur isolated in patients with both conditions. In children, the occurrence of red ear during migraine episodes is more common. However, the frequency of red ear associated with migraine is rare, it does not occur in all headache episodes. ${ }^{6}$ The frequency of attacks also varies and is similar to the frequency of migraine in migraine patients. ${ }^{4,8}$ The attacks may occur with a frequency ranging from several a day to a few per year. ${ }^{9}$ Duration may vary from minutes to hours, most lasting between 30 minutes to 1 hour., 5

In idiopathic cases, the main triggering factors for attacks are temperature changes and tactile stimuli, as touch or friction, mostly. Nevertheless, episodes could also occur spontaneously. ${ }^{9}$ Attacks may be bilateral, unilateral and alternate sides. Ipsilaterality and duration are significant implications for the understanding that RES is directly related with migraine. When red ear occurs alone, it has been proposed that it may be an episode of an "acephalgic migraine". ${ }^{4}$ The occurrence of bilateral attacks of red ear is more common in children with migraine. ${ }^{6}$

In most cases, pain accompanied by ear erythema are the only symptoms. Other autonomic phenomena may be present during the attacks, as in the case presented in this investigation. The most common autonomic phenomena are hyperhidrosis, edema and tearing. Such phenomena, added to the short duration of episodes, suggest that RES may be a form of Trigeminal Autonomic Cephalalgia. 9,10 Notwithstanding, the association between RES and migraine is more common in literature than TAC's. ${ }^{2,4,6} \mathrm{~A}$ review of literature including about 60 cases, showed that $55 \%$ of patients with primary RES suffer from migraine. ${ }^{7}$

\section{Physiopathology}

According to the proposed pathophysiological hypothesis for the frequent association between migraine and RES, it is plausible that both conditions share pathophysiological mechanisms. It has been proposed that, during migraine attacks, a trigemino vascular activation results in vasodilation by direct release of vasodilator peptides such as substance P, CGRP and others. ${ }^{4}$ This activation would explain the pain that may extend beyond the trigeminal innervation territory due to the overlap between trigeminal and upper cervical spinal nerves in the trigemino-cervical complex. This anatomical relationship may explain the occurrence of neck pain in patients with migraine. ${ }^{11}$

Another mechanism that might account is the sensory innervation promoted by the trigeminal nerve in the external carotid artery. Ergo, an activation in its nucleus could trigger an antidromic impulse and release of vasodilating substances in this vessel responsible for the blood supply to the ear. ${ }^{4}$ In addition, some migraine episodes may result in facial pallor due to an imbalance between sympathetic and parasympathetic vasomotor innervation.

When the red ear phenomenon appears during migraine, it is possible to observe facial vasoconstriction and ear vasodilation, indicating the activation of different neuronal modules. ${ }^{5}$ The same authors showed that RES preceding migraine attack, such as an aura phenomenon, may indicate the syndrome has an isolated neuronal system which can be activated during the migraine.

On the other hand, when RES occurs after migraine, some authors believe that it is an atypical migraine with characteristics of migraine and other primary headaches, and they justify it by the modular theory. ${ }^{12}$ This theory proposes that a group of neurons called modules are anatomically linked to one another and become activated in a manner characteristic for the individual. The theory could also explain why not all migraine attacks are accompanied by RES (before, during or after) even in patients suffering from both conditions. ${ }^{6}$ Even when the attacks are not time related, the history of migraine should be investigated. Raieli et al. ${ }^{8}$ showed that $50 \%$ of isolated RES cases have also a history of migraine.

\section{The association between RES and migraine}

When both conditions are related, the red ear could be considered a sign of hyper parasympathetic activation via trigeminal-autonomic reflex during migraine, which leads to consider RES a phenomenon related to migraine. ${ }^{9}$ This activation is also consistent with the phenomenon of red ear in primary headaches with autonomic involvement and could explain the existence of autonomic features in patients with migraine. ${ }^{10}$ Furthermore, mechanisms in migraine are not only capable of stimulating the trigemino-autonomic reflex, but can also stimulate a cervical autonomic reflex resulting in the RES. ${ }^{10}$

In addition to the occurrence of both disorders in the same patient, what supports the hypothesis that RES shares pathophysiological mechanisms in common with primary headaches is the resolution or a reduction in frequency and severity of RES. It occurs when treatment is initiated for the coexisting migraine. Beta-blocker and calcium channel blocker (flunarizine and nimodipine) showed a good response in both disorders. 3,9 However, because there are many potential causes, there is not a single treatment suggested, and many patients are treatment resistant. 
The importance to know the pathophysiology is to identify a therapeutic target and treatment. Several drugs for headache and pain were used in patients with this syndrome. Nonetheless, it is considered refractory to several drugs and varies from person to person. ${ }^{6}$

More studies with the adult population are needed to identify the prevalence of RES. The relationship between RES and primary headaches, mainly migraine, is strongly suggested. Thus, RES should be investigated in all patients with migraine.

Author contributions: The authors contributed equally in the preparation of the manuscript.

Conflict of interest: There is no conflict of interest.

Funding: The authors received no financial support for the research, authorship, and/or publication of this article.

Juliana Baleki Borri

https://orcid.org/0000-0003-2590-6426

Hilton Mariano da Silva Junior

https://orcid.org/0000-0002-9778-9946

\section{References}

1. Lance JW. The red ear syndrome. Neurology 1996;47(3):617-620 Doi:10.1212/wnl.47.3.617

2. Donnet $A$ and Valade $D$. The red ear syndrome. J Neurol Neurosurg Psychiatry 2004;75(7):1077 Doi:10.1136/jnnp.2003.030742

3. Purdy RA and Dodick DW. Red ear syndrome. Curr Pain Headache Rep 2007;11(4):313-316 Doi:10.1007/ s1 1916-007-0210-8

4. Raieli V, Monastero R, Santangelo G, Eliseo GL, Eliseo $M$ and Camarda $R$. Red ear syndrome and migraine: report of eight cases. Headache 2002;42(2):147-151 Doi:10.1046/i.1526-4610.2002.02033.x

5. Raieli V, Pandolfi E, La Vecchia M, Puma D, Calò $A$, Celauro $A$ and Ragusa D. The prevalence of allodynia, osmophobia and red ear syndrome in the juvenile headache: preliminary data. I Headache Pain 2005;6(4):271-273 Doi:10.1007/s10194-0050205-y

6. Raieli V, Compagno A, Brighina F, La Franca G, Puma D, Ragusa D, . . . D'Amelio M. Prevalence of red ear syndrome in juvenile primary headaches. Cephalalgia 2011;31(5):597-602 Doi: $10.1177 / 0333102410388437$

7. Ryan S, Wakerley BR and Davies P. Red ear syndrome: a review of all published cases (19962010). Cephalalgia 2013;33(3):190-201 Doi: $10.1177 / 0333102412468673$

8. Raieli V, Compagno A and D'Amelio M. Red Ear Syndrome. Curr Pain Headache Rep 2016;20(3):19 Doi:10.1007/s 1 1916-016-0547-y

9. Lambru G, Miller $S$ and Matharu MS. The red ear syndrome. J Headache Pain 2013;14(1):83 Doi: 10.1186/1129-2377-14-83

10. Al-Din AS, Mir R, Davey R, Lily O and Ghaus N. Trigeminal cephalgias and facial pain syndromes associated with autonomic dysfunction. Cephalalgia 2005;25(8):605-61 1 Doi: $10.1111 /$ j.14682982.2005.00935.x

11. Marinis $M$ and Accornero N. Recurrent neck pain as a variant of migraine: description of four cases. $J$ Neurol Neurosurg Psychiatry 1997;62(6):669-670 Doi: 10.1136/innp.62.6.669

12. Young WB, Peres MF and Rozen TD. Modular headache theory. Cephalalgia 2001;21(8):842-849 Doi:10.1046/j.1468-2982.2001.218254.x 